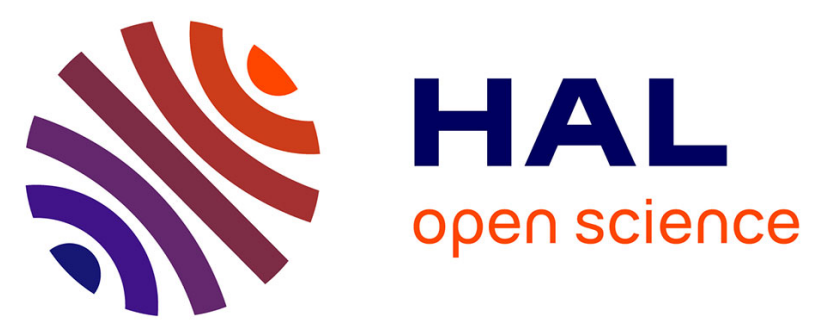

\title{
Neural oscillations track natural but not artificial fast speech: Novel insights from speech-brain coupling using MEG
}

Ana Sofía Hincapié Casas, Tarek Lajnef, Annalisa Pascarella, Hélène Guiraud-Vinatea, Hannu Laaksonen, Dimitri Bayle, Karim Jerbi, Véronique

Boulenger

\section{To cite this version:}

Ana Sofía Hincapié Casas, Tarek Lajnef, Annalisa Pascarella, Hélène Guiraud-Vinatea, Hannu Laaksonen, et al.. Neural oscillations track natural but not artificial fast speech: Novel insights from speechbrain coupling using MEG. NeuroImage, 2021, 244, pp.118577. 10.1016/j.neuroimage.2021.118577 . hal-03373459

\author{
HAL Id: hal-03373459 \\ https://hal.science/hal-03373459
}

Submitted on 11 Oct 2021

HAL is a multi-disciplinary open access archive for the deposit and dissemination of scientific research documents, whether they are published or not. The documents may come from teaching and research institutions in France or abroad, or from public or private research centers.
L'archive ouverte pluridisciplinaire HAL, est destinée au dépôt et à la diffusion de documents scientifiques de niveau recherche, publiés ou non, émanant des établissements d'enseignement et de recherche français ou étrangers, des laboratoires publics ou privés. 


\title{
Neural oscillations track natural but not artificial fast speech: Novel insights from speech-brain coupling using MEG
}

\author{
Ana Sofía Hincapié Casas a,b, Tarek Lajnef a,1, Annalisa Pascarella ${ }^{\mathrm{c}, 1}$, Hélène Guiraud-Vinatea ${ }^{\mathrm{d}}$, \\ Hannu Laaksonen ${ }^{\text {d,e }}$, Dimitri Bayle ${ }^{f}$, Karim Jerbi ${ }^{\text {a,e,g,h,2, }}$, Véronique Boulenger ${ }^{\text {d,2,* }}$ \\ ${ }^{a}$ Cognitive and Computational Neuroscience Lab, Psychology Department, University of Montreal, Montreal, Quebec H2V 2S9, Canada \\ ${ }^{\mathrm{b}}$ Department of Neurology and Neurosurgery, Montreal Neurological Institute, Faculty of Medicine, McGill University, Montreal, Quebec H3A 2B4, Canada \\ ${ }^{\mathrm{c}}$ Institute for Applied Mathematics Mauro Picone, National Research Council, Roma 00185, Italy \\ ${ }^{d}$ Dynamics of Language laboratory, UMR5596 CNRS/University of Lyon, Lyon 69007, France \\ ${ }^{\mathrm{e}}$ Lyon Neuroscience Research Center, DyCog team, INSERM U1028 / CNRS UMR5292, Bron 69500, France \\ ${ }^{\mathrm{f}}$ LICAE Lab, UPL, Paris Nanterre University, Nanterre 92000, France \\ ${ }^{g}$ MILA (Québec Artificial Intelligence Institute), QC H2S 3H1 Montréal, Québec, Canada \\ ${ }^{\mathrm{h}}$ MEG Center, University of Montreal, Montreal, Quebec H2V 2S9, Canada
}

\section{A R T I C L E I N F O}

\section{Keywords:}

Speech processing

Neural entrainment

Syllable rate

Natural speech

Neuronal Oscillations

Cortico-acoustic coupling

Magnetoencephalography (MEG)

\begin{abstract}
A B S T R A C T
Neural oscillations contribute to speech parsing via cortical tracking of hierarchical linguistic structures, including syllable rate. While the properties of neural entrainment have been largely probed with speech stimuli at either normal or artificially accelerated rates, the important case of natural fast speech has been largely overlooked. Using magnetoencephalography, we found that listening to naturally-produced speech was associated with cortico-acoustic coupling, both at normal ( $\sim 6$ syllables/s) and fast ( $\sim 9$ syllables $/ s)$ rates, with a corresponding shift in peak entrainment frequency. Interestingly, time-compressed sentences did not yield such coupling, despite being generated at the same rate as the natural fast sentences. Additionally, neural activity in right motor cortex exhibited stronger tuning to natural fast rather than to artificially accelerated speech, and showed evidence for stronger phase-coupling with left temporo-parietal and motor areas. These findings are highly relevant for our understanding of the role played by auditory and motor cortex oscillations in the perception of naturally produced speech.
\end{abstract}

\section{Introduction}

Understanding how the brain parses the continuous speech stream into smaller linguistic units remains a challenging issue, especially given the large variability of the speech acoustic signal. In this regard, the (quasi-)rhythmicity of speech is fundamental as it allows the listener's cognitive system to make predictions about the incoming signal, thus helping speech segmentation and comprehension (Peelle and Davis, 2012). Neurocognitive models of speech perception assign a key functional role to ongoing neural oscillations in the tracking of speech (Ghitza, 2011; Giraud and Poeppel, 2012; Poeppel, 2003). By aligning to speech at multiple timescales, brain oscillatory activity in theta $(\sim 4-7 \mathrm{~Hz})$ and delta $(\sim 1-3 \mathrm{~Hz})$ frequency bands would parse the acoustic flow into syllable- and word-sized packets, respectively. Amplitude of gamma oscillations, nested in the theta phase, would in parallel encode phonemic features within theta-chunks. These units may then be integrated hierarchically for higher-order linguistic processes. Convincing evidence from electro- and magnetoencephalography (EEG/MEG) revealed coupling between theta oscillations in the auditory cortex and the slow modulations $(2-8 \mathrm{~Hz})$ in speech amplitude envelope (Doelling et al., 2014; Park et al., 2015; Peelle et al., 2013; Gross et al., 2013), which are inherently tied to syllable production (Chandrasekaran et al., 2009) and are crucial for speech intelligibility (Rosen, 1992). Interestingly, auditory cortex oscillations are therefore able to track the speech input in a frequency range which coincides with the average syllable rate of speakers across languages (Pellegrino et al., 2011).

Speech rate can however substantially vary within and between speakers and contexts, requiring the listener to rapidly adapt. Surprisingly, only a few studies so far examined brain-to-speech coupling in the case of speech rate variations, and we know little about the spatial and frequency dynamics of cortical oscillations involved in process-

\footnotetext{
* Corresponding author.

E-mail address: veronique.boulenger@cnrs.fr (V. Boulenger).

1 These authors equally contributed to this work.

${ }^{2}$ Karim Jerbi and Véronique Boulenger equally contributed to this work.
} 
ing naturally accelerated speech. Most of previous studies used timecompressed speech, where the duration is artificially reduced but the spectral content is kept intact (Hertrich et al., 2012; Kösem et al., 2018b). Results showed that brain-to-speech coupling occurs for moderately time-compressed, intelligible speech but not for unintelligible stimuli with a syllable rate exceeding the upper limit of the neural theta band (Ahissar et al., 2001; Bosker and Ghitza, 2018; Doelling et al., 2014; Ghitza, 2012). Accordingly, the efficiency of speech decoding may depend on the capacity of neural theta oscillations to remain in sync with the syllable rate. This said, one EEG study (Pefkou et al., 2017) reported cortical coupling to the syllabic structure of time-compressed speech up to $14 \mathrm{~Hz}$, even for poorly understood sentences. This suggests that neural oscillations are able to align to the incoming speech signal at higher frequencies to match its temporal structure, at least for artificial acceleration (see also Lizarazu et al., 2019) and even if speech is not fully intelligible (but see Riecke et al., 2018).

Taken together, previous studies of neural coupling to fast rate speech have mainly focused on artificially compressed stimuli and have led to heterogenous and partly contradictory results. In fact, although insightful, studying the perception of time-compressed speech may not provide the best model of how neural oscillations handle natural changes in speech rate. This question has been largely overlooked in the literature, yet it seems crucial given the subtle differences between naturally-produced fast speech and artificially accelerated speech. By contrast to time-compressed speech, reduction of acoustic cues indeed operates non-linearly when we naturally speak faster, partly due to articulatory restrictions (Janse, 2004). Natural fast speech also undergoes a series of spectro-temporal changes resulting in increased processing load for the listener as compared to time-compressed speech (Guiraud et al., 2018; Janse, 2004). Uttering speech at a fast rate decreases the spatial magnitude of articulatory movements and enhances coarticulation (i.e., increased gestural overlap), which can even lead to whole segment suppression (Berry, 2011). Accordingly, the listener's auditory system has to adjust not only to a shortened (as in timecompressed speech) but also spectro-temporally degraded signal for efficient decoding. Although adaptation to naturally accelerated speech has been reported behaviourally (Adank and Janse, 2009), the underlying brain oscillatory dynamics remain largely underinvestigated. More generally, and to the best of our knowledge, no study to date has directly compared neural entrainment to speech using either natural fast rate speech or artificially accelerated stimuli. Such a comparison may elucidate whether using artificial acceleration of speech accurately captures the brain mechanisms at play during perception of natural speech rate changes.

Here we address this question using an unprecedented MEG experiment where we compare the modulations of cortico-acoustic tracking patterns induced by normal and fast speech, generated either naturally or using time compression. Although seemingly subtle, the distinction we address is of fundamental importance to better understand how our brains track and encode the spectro-temporal changes we encounter in daily communication. Furthermore, revealing differences in the neural processing of naturally versus artificially accelerated speech could be key to improving oscillatory models of speech perception. Based on previous work (Lizarazu et al., 2019; Pefkou et al., 2017), one would expect that increases in speech rate would be associated with upward shifts in cortico-acoustic coupling frequency, matching the change in syllable rate, and that this would occur both for natural and artificial acceleration of the stimuli. However, given the articulatory changes elicited by natural acceleration (Berry, 2011) and the tight relationship between speech perception and production processes (Hickok and Poeppel, 2007; Hickok et al., 2011; Pulvermüller and Fadiga, 2010), the parsing process may specifically engage sensorimotor mechanisms during natural fast speech perception. As a result, we expect neural coupling to occur not only in auditory but also in motor regions and importantly, that this motor resonance may be stronger for naturally than for artificially accelerated speech.
To test these assumptions, we sought to unravel the oscillatory brain signature of speech naturally produced at a normal or fast rate, and of time-compressed speech. We investigated whether neural oscillations in auditory and motor cortex, recorded with MEG, align to syllable rate when it is naturally accelerated and how this compares to artificially manipulated speech. Speakers can naturally slow down or speed up their syllable rate outside the limits of this range. Examining cortical tracking of speech rate variations in frequency ranges that specifically match the temporal structure of heard speech, and not in the canonical theta band, therefore appears as a more straightforward approach (Keitel et al., 2018). Accordingly, we assessed cortical coupling to the envelope of normal and fast rate speech at the source level at two frequencies of interest, identified as peaks in the respective power spectra of the speech signals. The results of the speech-brain coupling were used to further examine the oscillatory network dynamics using seed-based phase-coupling analyses. Finally, by including amplitude-modulated noise control stimuli, we additionally investigated whether cortico-acoustic coupling depends on the presence of fine spectro-temporal information in the stimuli or simply reflects brain responses to low-level rhythmic acoustic cues (Ding and Simon, 2014).

\section{Materials and methods}

\subsection{Participants}

Twenty-four French native speakers participated in the study after providing informed consent (14 females, mean age 23 years old, range 18-45 years). All participants were right-handed (mean score at the Edinburgh handedness inventory $=94$ ) (Oldfield, 1971) and reported normal hearing together with no history of neurological or psychiatric disorder. The protocol conformed to the Declaration of Helsinki and was approved by the local ethical committee (Comité de Protection des Personnes Lyon Sud-Est II; ID RCB: 2012-A00857-36). Participants received monetary compensation for their participation.

\subsection{Stimuli}

We created 288 meaningful sentences (7-9 words) following the same syntactic structure: Determiner - Noun 1 - Verb - Determiner - Noun 2 - Preposition - Determiner - Noun 3 (e.g., "Sa fille déteste la nourriture de la cantine" / His-daughter hates the food at the canteen). Sentences were recorded in a sound-attenuated booth by a French native professional theater actor who was able to produce sentences at the required fast rate while remaining intelligible. We recorded each sentence twice ( $44.1 \mathrm{kHz}$, mono, 16 bits) using ROCme! software (Ferragne et al., 2012), first at a normal and then at a fast rate. The procedure was the following: the sentence was first displayed on a computer screen in front of the speaker who was instructed to silently read it and to subsequently produce it aloud as a declarative statement at his normal speech rate. The speaker then produced the sentences at a fast rate (i.e. as fast as possible while remaining intelligible) using the same procedure (i.e. no external pacing was imposed).

We calculated the durations of the $2 \times 288$ sentences and the number of actually produced syllables for each sentence with Praat software (Boersma and Weenik, 2012). The mean syllable rate was 6.76 syllables/s (SD 0.57) for natural normal rate sentences and 9.15 syllables/s (SD 0.60) for natural fast rate sentences. This led to an overall fast-tonormal ratio of 0.74 (i.e. speed-up factor of 1.35). Subsequently, we computed time-compressed sentences by digitally shortening them with a PSOLA (Pitch Synchronous Overlap and Add) algorithm (Moulines and Charpentier, 1990), as implemented in Praat. We obtained compression rates for each sentence: we matched each individual time-compressed sentence in terms of syllable rate to its equivalent natural fast item. This artificial compression corresponds to a re-synthesis of the natural normal rate stimulus, changing only its temporal structure. For the total of 864 sound files ( $288 \times 3$ speech rate variants), we applied an $80 \mathrm{~Hz}$ 
high-pass filter, and smoothed the amplitude envelope sentence-initially and finally. We then peak normalized the intensity of the sound files.

The intelligibility of the sentences used as stimuli in this study was verified with an online experiment conducted in a group of 42 French native participants ( 28 females, mean age 31 years old \pm 7 ) who did not take part in the MEG experiment. They were asked to listen to each of the sentences once and rate their intelligibility on a 4-point scale (1: not intelligible at all; 2: little intelligible; 3: rather intelligible; 4: perfectly intelligible). For $25 \%$ of the trials, a true/false test statement was proposed to assess comprehension as well as to monitor attention to the stimuli. A repeated-measure analysis of variance showed a significant expected effect of speech rate $\left(\mathrm{F}(2,82)=75.53, p<.001, \eta^{2}=0.648\right)$, with higher mean intelligibility scores for normal rate sentences $(3.81 \pm 0.50)$ than for natural fast $(3.01 \pm 0.58, p<.001$ Bonferroni post-hoc test) and time-compressed speech $(3.38 \pm 0.55, p<.001)$. The two accelerated conditions also significantly differed from each other $(p<.001)$. This is in line with previous studies showing an advantage of artificial compression over natural acceleration in speech comprehension (e.g., Guiraud et al., 2018; Janse, 2004). Importantly, the results reveal that natural fast sentences were still intelligible despite natural acceleration. This was confirmed by analysis of responses to test statements showing high $\%$ of correct responses in the natural fast condition ( $97 \%$ vs $98.3 \%$ for normal rate and $98 \%$ for time-compressed speech).

Finally, for each of the 288 normal rate and 288 fast rate sentences, we created amplitude-modulated noise stimuli using the amplitude envelopes of the sentence material. These stimuli served as control nonspeech conditions. We also used 48 filler sentences (different from the experimental stimuli but with the same syntactic structure, either at a normal rate, natural fast rate, or time-compressed) in which we added beep-sounds at the end and to which the participants had to respond during the experiment (see MEG data acquisition and task design).

We divided the total number of stimuli into two experimental lists, each including 288 sentences ( 96 normal rate, 96 natural fast rate and 96 time-compressed), 192 amplitude-modulated noise stimuli (96 with normal rate envelope and 96 with fast rate envelope) and 48 filler sentences. Each stimulus appeared in each rate condition across all participants but only once per list (to avoid repetition effects).

\subsection{MEG data acquisition and task design}

Participants were comfortably sitting in a sound-attenuated, magnetically-shielded recording room with a screen in front of them. We presented stimuli binaurally through air-conducting tubes with foam insert earphones (Etymotic ER2 and ER3). Prior to the MEG recording, we determined participants' auditory detection thresholds for each ear with a $1 \mathrm{~min}$ pure tone of $44 \mathrm{kHz}$; the level was then adjusted so that we presented the stimuli at $50 \mathrm{~dB}$ Sensation Level with a central position (stereo) with respect to the participant's head.

During the experiment, participants attentively listened to all stimuli from one of the two experimental lists while looking at a fixation cross at the center of the screen. Their task was to detect beep-sounds embedded in filler sentences by pressing a button (response button Neuroscan, Pantev) with their left index finger. Participants detected $100 \%$ of these trials, which we excluded from subsequent analysis. We pseudo-randomly presented all stimuli in 8 blocks of 66 trials allowing for short breaks. A training phase with 5 sentences (different from experimental stimuli) preceded the actual experiment. Each trial started with the appearance of a fixation cross which remained on the screen throughout the duration of the trial. An auditory stimulus (sentence or amplitude-modulated noise) was delivered $1500 \mathrm{~ms}$ after trial onset. Each trial was followed by an inter-trial interval (gray screen) of $1250 \mathrm{~ms}$. We instructed the participants to attentively listen to the presented stimuli. In the case of filler stimuli, they had to press the response button as quickly as possible. To maintain the participants' attention throughout the experiment, we informed them there would be questions about the content of the sentences at the end of the experiment. We used Presentation software (Neurobehavioral Systems) to run the experiment.

We recorded brain activity of the 24 participants using a 275-channel whole-head MEG system (CTF OMEGA 275, Canada) at $1200 \mathrm{~Hz}$ sampling rate. We placed three fiducial coils (nasion, left and right preauricular points) on each participant to determine head position within the MEG helmet. Four electrooculographic (EOG) electrodes also allowed to record horizontal and vertical eye movements. We monitored reference head position before each of the 8 experimental blocks and tracked head movements throughout the experiment using continuous head position identification (HPI).

\subsection{Data analysis}

We performed all analyses using custom written Matlab scripts (Mathworks Inc., MA, USA) and the Fieldtrip toolbox (Oostenveld et al., 2011). Fig. 1 describes the general methodology. The following section describes the processing of (1) speech recordings, (2) MEG data, (3) source reconstruction, (4) spectral power and cortico-acoustic coherence estimation, and (5) statistical analyses.

\subsubsection{Speech recordings (reference signals)}

We computed the amplitude envelope of the speech signal following the methodology of Peelle and colleagues (Peelle et al., 2013). We first rectified the signal (full wave rectification) and then filtered it using a Butterworth low-pass filter $(30 \mathrm{~Hz}$, fifth order Butterworth filter, zero-phase digital filtering). For the cortico-acoustic coupling analysis, we selected speech envelope segments between 200 and 1000 ms poststimulus onset (equal to the time of interest of the functional MEG data). We computed the spectral power for all sentence envelopes for each condition and identified the central frequencies of the prominent rhythmic components, using the FOOOF algorithm (fitting a parameteric model to the power spectral densities PSD) and subtracting the $1 / \mathrm{f}$ (i.e. aperiodic) component (Haller et al., 2018). The main peaks in the speech PSD were found at $6.35 \mathrm{~Hz}$ for normal rate, $8.91 \mathrm{~Hz}$ for natural fast rate and $8.61 \mathrm{~Hz}$ for time-compressed speech (Fig. 2, see supplementary Table S1 and Fig. S1 for details), which closely match the mean syllable rates calculated with Praat (6.76 syllables/s (SD 0.57) for natural normal rate and 9.15 syllables/s (SD 0.60) for natural fast and timecompressed speech). The frequency peaks identified in the spectra of the speech signals using the FOOOF method were used to define the frequencies of interest for the coherence analysis, namely $6.25( \pm 1 \mathrm{~Hz})$ and $8.75( \pm 1 \mathrm{~Hz})$ for the normal and fast speech rates respectively.

\subsection{2. $M E G$ data}

We first segmented the MEG data into periods of $3 \mathrm{~s}$ (from $1 \mathrm{~s}$ before stimulus onset to $2 \mathrm{~s}$ after onset) for preprocessing. We rejected data segments contaminated by eye blinks, heartbeat and muscle artefacts using a standard semiautomatic procedure available in the Fieldtrip toolbox as follows. First, we applied Notch filters at 50, 100 and $150 \mathrm{~Hz}$ to suppress electrical line noise (using ft_preprocessing function, with default settings), we then re-sampled the data to $300 \mathrm{~Hz}$ and rejected the deviant trials from visual inspection. Second, we detected and rejected EOG artifacts, jumps and muscle artifacts and visually double-checked the trials. Finally, we performed an Independent Component Analysis (ICA) to correct for electrocardiographic (ECG) artifacts as well as to check for residual EOG artifacts. For each trial, we defined the speech encoding period (i.e. active period) as the time from $200 \mathrm{~ms}$ to $1000 \mathrm{~ms}$ post-stimulus onset, and the baseline from $1000 \mathrm{~ms}$ to $200 \mathrm{~ms}$ before onset.

\subsubsection{Source reconstruction}

We acquired the T1-weighted structural MRIs (MRI 1.5 T, Siemens AvantoFit) of 23 of the 24 participants after the MEG study. We aligned each MRI to the MEG coordinate system using the localization coils 


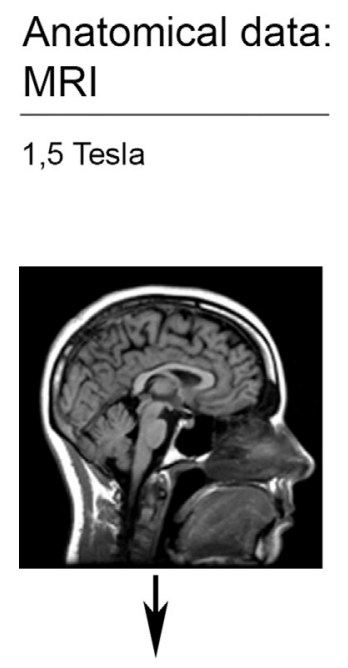

Anatomical data:
MRI

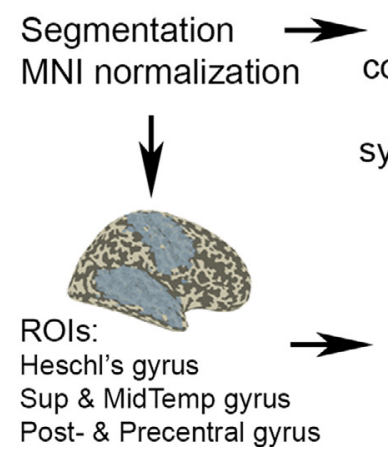

\section{Functional data: MEG}

24 right-handed subjects Normal hearing French native speakers

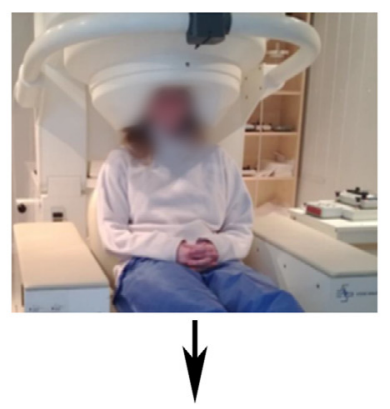

Source power and cortico-acoustic coherence at frequencies centered on syllable rate (DICS beamformer)

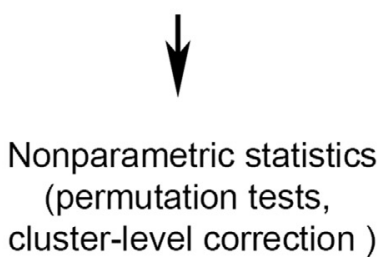

Audio data: Speech (sentences)

French native male speaker (professional actor)

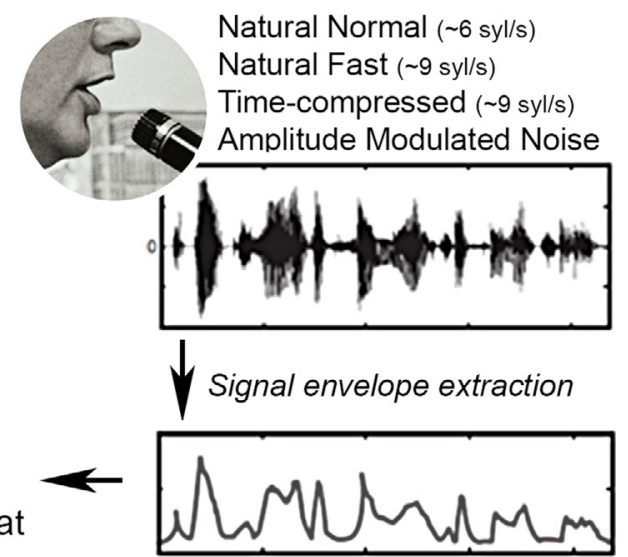

\section{Neural entrainement} at syllable rate?

Fig. 1. Overview of the cortico-acoustic coupling analysis. Computation of cortico-acoustic coherence required three inputs: the reference signal, namely the audio recordings; the anatomical data, i.e. the participants' MRI; and the functional data, i.e. MEG recordings. We used the amplitude envelope of speech as the reference signal to investigate cortical alignment at the syllable rate. We segmented each participant's MRI and then constructed the source space based on a warped Montreal Neurological Institute (MNI) anatomical grid template, which we used for group analysis. After preprocessing the individual MEG recordings, we computed the source modeling and cortico-acoustic coupling using the Dynamical Imaging of Coherent Sources (DICS) beamformer (Gross et al., 2001). Lastly, for statistical analysis we applied non-parametric randomization, cluster-based permutation statistical tests across participants for each frequency of interest and condition in five bilateral regions-of-interest (ROIs). We achieved the statistical assessment of cortico-acoustic coherence by comparison to two control conditions: coherence obtained either using surrogate data (trial shuffling) or using pre-stimulus data.

marked on the MRIs and the interactive alignment option in the Fieldtrip toolbox. We segmented each MRI and then computed each subject's head model and source space using Fieldtrip. We used the single-shell as volume conductor model (Nolte, 2003). We constructed each subject's source space based on a warped MNI template grid. This way, each location on the template grid corresponds to homologous grid points across subjects, which allows us to run group-level analyses in a common source level. Source estimation was done with a volumetric source-model (3-dimensional template grid with 8693 vertices) and using Dynamical Imaging of Coherent Sources (DICS), a frequency-domain beamformer (Gross et al., 2001).

\subsubsection{Spectral power and cortico-acoustic coherence estimation}

We estimated source power and cortico-acoustic coupling using DICS. This allowed us to assess cortico-acoustic coupling by computing coherence between the speech envelope and the reconstructed cortical activity. The magnitude squared coherence is defined as the linear correlation between two signals as a function of frequency. It is mathematically defined by:

$\operatorname{Coh}\left(r_{e f}, r_{c}, f\right)=\left|\operatorname{Cs}\left(r_{e f}, r_{c}, f\right)\right|^{2} /\left(\operatorname{Cs}\left(r_{e f}, r_{e f}, f\right) \operatorname{Cs}\left(r_{c}, r_{c}, f\right)\right)$

Where $\boldsymbol{r}_{e f}$ is the reference signal, namely the amplitude envelope of the speech signal; $\boldsymbol{r}_{\boldsymbol{c}}$ is the signal at each vertex of the anatomical grid estimated with DICS; $\boldsymbol{f}$ is the frequency bin and $\boldsymbol{C s}$ is the cross spectral density matrix.
We computed the cross spectral density (CSD) matrix using the multitaper FFT with Slepian tapers (i.e. Discrete Prolate Spheroidal Sequences, DPSS), at the frequency bins closest to the frequencies of interest, with $\pm 1 \mathrm{~Hz}$ of spectral smoothing. The identification of the frequencies of interest for the normal and fast conditions was based on the FOOOF analysis. Given the length of the data windows ( $0.8 \mathrm{~s})$, the coherence frequency bins closest to the peaks identified from the spectra of the speech signals (using FOOOF) turned out to be 6.25 and $8.75 \mathrm{~Hz}$ for the normal and fast speech conditions respectively.

In addition to computing cortico-acoustic coherence during the speech encoding period (200 to $1000 \mathrm{~ms}$ ), we also computed corticoacoustic coherence (a) for the baseline window ( -1000 to $-200 \mathrm{~ms}$ ) and (b) for shuffled data, as control conditions for statistical analysis. In the case of shuffled data, we randomly permuted the speech trial order (i.e. destroying the correspondence between the speech stimulus heard by the participant and the associated MEG data segment) before computing cortico-acoustic coherence. We repeated the shuffling procedure 100 times and averaged coherence across iterations for each participant, each condition, each node and frequency of interest. This led to 23 surrogate coherence values associated with 23 true coherence values, for each node, frequency and condition.

\subsubsection{Cortico-cortical connectivity analyses}

To quantify task-based modulations of cortico-cortical interaction patterns, we computed seed-based connectivity via weighted Phase Lag 
Power spectral density of speech signals (Full model fit using FOOOF)

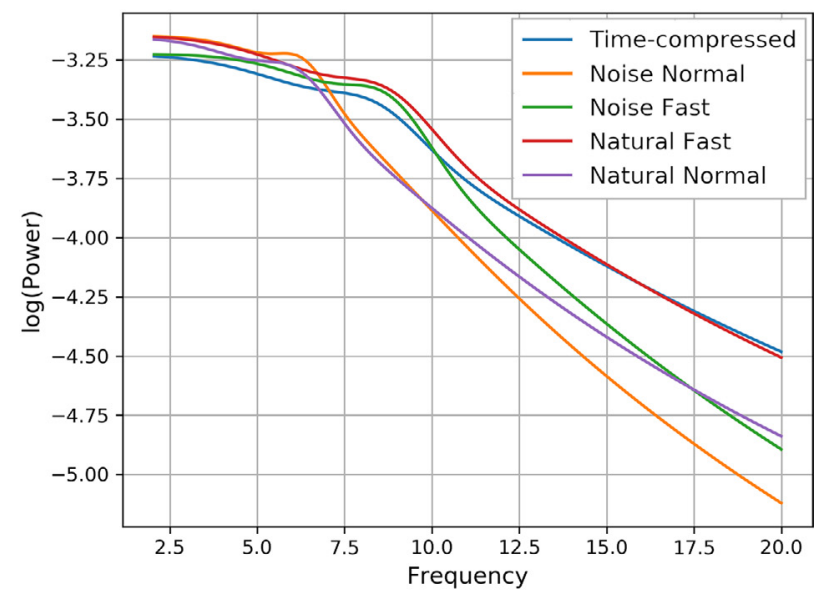

B

Periodic components of speech signals (removing aperiodic components from full model)

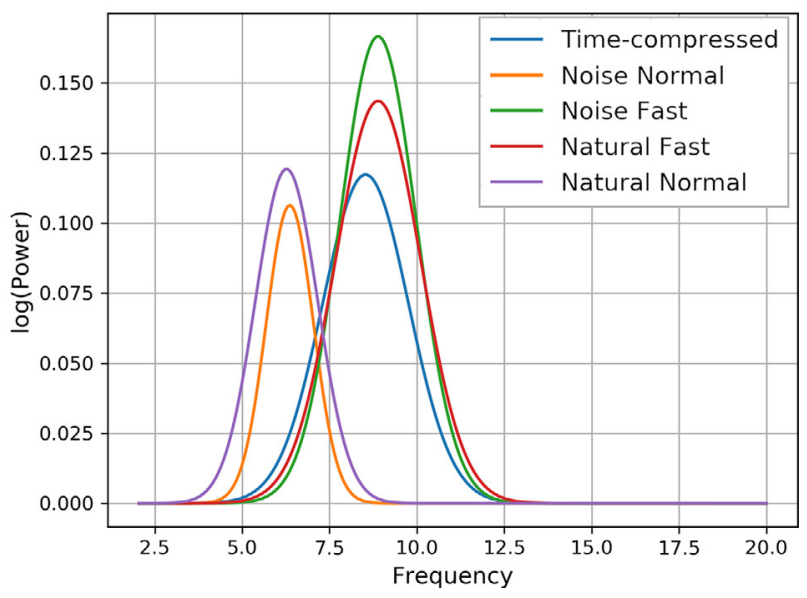

Fig. 2. Power spectra of the acoustic signal for the five conditions. (A) Power spectra of the acoustic signals' envelopes across the five experimental conditions: Natural Normal and Natural Fast correspond to naturally-produced speech at a normal (mean syllable rate 6.76 syllables/s) and fast rate (mean rate 9.15 syll/s), respectively. Time-compressed speech was compressed at the same syllable rate as in the natural fast rate condition (mean syllable rate $9.15 \mathrm{syll} / \mathrm{s}$ ). Noise Normal and Noise Fast correspond to noise stimuli modulated with the amplitude envelopes of normal rate and fast rate sentences, respectively. The depicted spectra represent parametric model fits of the PSD (Power Spectral Density), that consist of aperiodic and periodic components computed using the FOOOF algorithm (Haller et al., 2018) (B) Periodic components of the full model shown in (A), i.e. after removal of the aperiodic (the so-called $1 / \mathrm{f}$ ) component of the spectra. The spectral power peaks in the speech stimuli for each condition occur at frequencies that match the corresponding mean syllable rates calculated using the Praat software (see Supplementary Table S1 and Supplementary Fig. S1 for details).

Index (wPLI). For each participant, we computed the wPLI connectivity matrix in the frequency band $\sim 8.75+/-1 \mathrm{~Hz}$ for three distinct conditions: time-compressed, natural fast rate and normal rate speech. We chose wPLI because of its relative insensitivity to linear mixing effects compared to other metrics such as coherence or phase-locking value (Palva et al., 2018; Vinck et al., 2011).

\subsubsection{Statistical analyses}

We conducted group statistical analysis for the 23 participants (out of 24) with individual MRI. As control conditions, we used shuffled data and the baseline period for cortico-acoustic coherence, and the baseline for source power analysis. We compared the speech encoding period to each control condition applying non-parametric Monte-Carlo randomization, dependent-samples T-test statistics using FieldTrip. We corrected for multiple comparisons using 'maxsum' cluster-based correction and used a statistical significance threshold of 0.05 (Maris and Oostenveld, 2007). In brief, the cluster-based correction we implemented operates by first detecting vertices whose t-values exceed a given threshold (we used 0.05) and then grouping these vertices into clusters based on spatial agency. The cluster-level statistics are then computed as the sum of the t-values within each cluster, and the maximum of all the sums is taken as the cluster-based test statistic (i.e. setting cfg.clusterstatistic to be 'maxsum'). The statistical significance is then assessed by generating a null-distribution of this test statistic (i.e., the maximum of the cluster-level summed t-values) calculated via 1000 random permutations of the condition labels. Because we hypothesized that brain-speech coherence increases compared to control conditions, we used one-sided tests for the coherence analyses. However, two-sided tests were used when we explored task-based differences in spectral power, since we tested for both increases and decreases of mean power. Furthermore, and in line with several previous studies on neural entrainment to speech (Keitel et al., 2017; Lizarazu et al., 2019; Peelle et al., 2013), statistical analyses were performed on a pre-defined source space, made up of multiple anatomical regions of interest (ROIs). These were determined using the Automated Anatomical Labeling (AAL) atlas (TzourioMazoyer et al., 2002). For coherence analyses, we chose ten ROIs (five in each hemisphere) based on neurocognitive models (Giraud and Poeppel, 2012; Hickok and Poeppel, 2007) and neuroimaging data on speech perception (Evans and Davis, 2015; Okada et al., 2010; Osnes et al., 2011). The ROIs consisted of Heschl's gyrus, superior temporal gyrus, middle temporal gyrus, precentral and postcentral gyri (bilaterally). For the analysis of direct contrasts between speech conditions, we additionally defined the motor/articulatory cortex ROI (a) in a data-driven manner, based on the location of the speech-brain coherence peak found at $\sim 8.75 \mathrm{~Hz}$, and (b) following previous reports identifying articulatory cortex (Brown et al., 2009; Glanz Iljina et al., 2018). This articulatory ROI was selected as all the voxels located within $8 \mathrm{~mm}$ from, respectively, two locations in MNI space at $(66,-4,17)$ and $(66,3,17)$. The multiple time series were always processed individually, and never averaged before coherence or spectral power analyses. Finally, we used the BioImage Suite software (www.bioimagesuite.org) (Lacadie et al., 2008; Papademetris et al., 2006) to identify the Brodmann areas detected as significant in the statistical analysis. As for the wPLI analyses, we used two key ROI as seeds: the right auditory ROI and the right articulatory/motor ROI. This choice was based on the results of the cortico-acoustic coupling analyses, as it was intended as an additional follow-up analysis. We contrasted the natural fast with (a) the time-compressed condition and (b) the normal rate condition using nonparametric Monte-Carlo randomization (10,000 randomizations) and dependent-samples one-sided T-test statistics using FieldTrip. Here too, we corrected for multiple comparisons using the 'maxsum' cluster-based correction (Maris and Oostenveld, 2007).

The data that support the findings of this study, as well as the codes used for analysis, are available from the corresponding author upon reasonable request.

\section{Results}

The ROI-based analysis using shuffled data as control revealed a shift in the frequency domain of cortical oscillations to align to the syllable rate of naturally-produced heard sentences. Significant increase of cortico-acoustic coherence for the normal rate condition was found at the frequency matching the mean normal syllable rate $(\sim 6.25 \mathrm{~Hz})$ (Fig. 3A). Crucially, for natural fast speech, coherence significantly increased at the frequency matching the mean fast syllable rate $(\sim 8.75 \mathrm{~Hz}$; Fig. 3B). The time-compressed speech condition did not show any signif- 
A

\author{
Cortico-acoustic Coherence \\ (Encoding vs Shuffled)
}

@ $6.25 \pm 1 \mathrm{~Hz}$

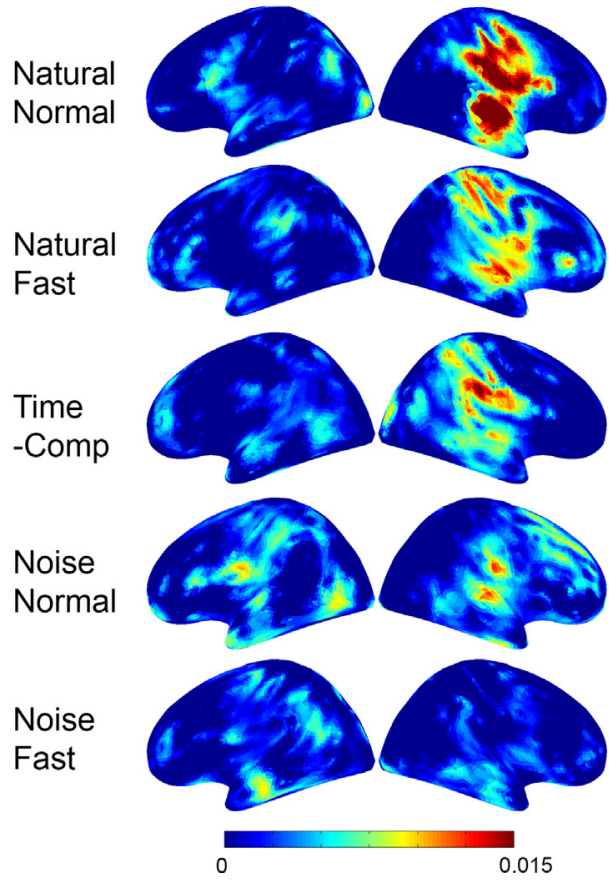

Statistical Maps $p<0.05$
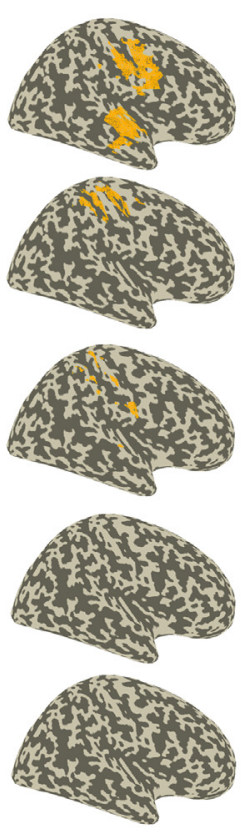

B

B Cortico-acoustic Coherence

(Encoding vs Shuffled)

@ $8.75 \pm 1 \mathrm{~Hz}$
Statistical Maps $p<0.05$
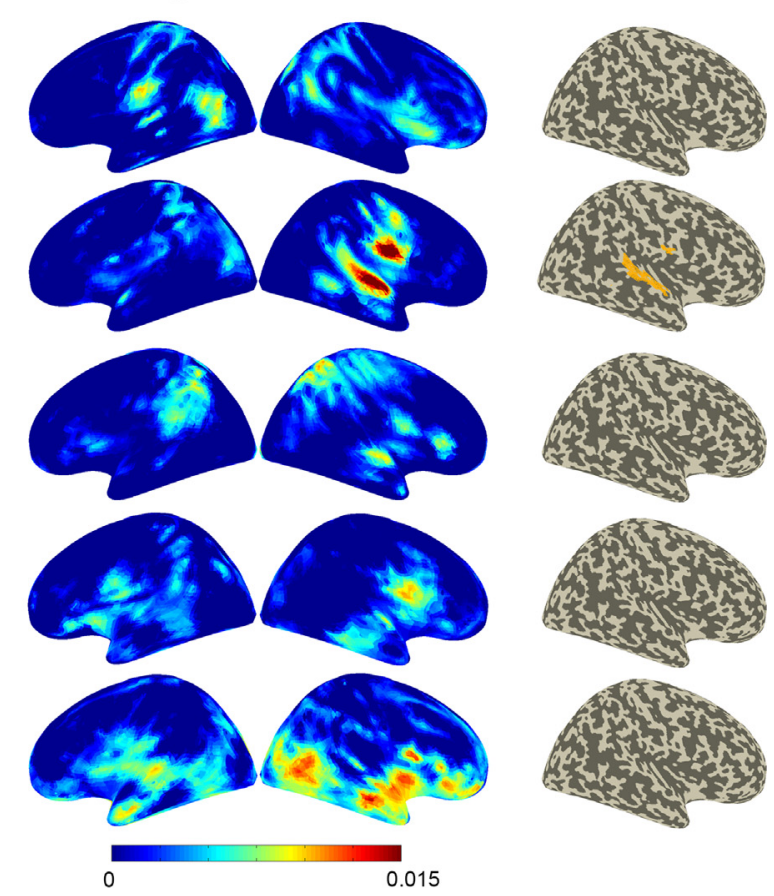

Fig. 3. Cortical tracking of speech at (A) $6.25( \pm 1 \mathrm{~Hz})$ and (B) $8.75( \pm 1 \mathrm{~Hz})$ matching the normal and fast speech rates respectively. Coherence maps between signal's amplitude envelope and neural oscillations in the active period (i.e. during stimulus presentation), as compared to surrogate data, together with statistical maps in the right hemisphere (corrected, $\alpha=0.05$; results were not significant in the left hemisphere) presented for the five conditions. Natural Normal $=$ naturally-produced normal rate speech; Natural Fast $=$ naturally-produced fast rate speech; Time-Comp $=$ artificially accelerated speech from normal rate sentences at the same rate as natural fast speech; Noise Normal = amplitude-modulated noise with the envelope of normal rate sentences; Noise Fast = amplitude-modulated noise with the envelope of natural fast rate sentences.

icant brain coupling at the corresponding frequency $(\sim 8.75 \mathrm{~Hz})$. Comparable patterns of cortico-acoustic coupling were obtained when coherence during speech encoding was compared to coherence computed for baseline data (Supplementary Fig. 2). As Fig. 3 shows, in both natural speech rate conditions, cortical tracking of speech envelope at the corresponding frequencies was seen in the primary auditory cortex (Brodmann Area BA 41), middle and superior temporal gyri (BAs 21/22), primary sensory (BA 1) and primary motor and premotor cortices (BAs $4 / 6$ ) of the right hemisphere (see also Supplementary Fig. 2). Note that we also found significant brain coupling in the right dorsal precentral gyrus (BA 4) for naturally and artificially accelerated speech at $\sim 6.25 \mathrm{~Hz}$ (normal rate frequency). By contrast, we did not find any significant increase in coherence for normal rate speech at the higher frequency $(\sim 8.75 \mathrm{~Hz})$. Neither of the two amplitude-modulated noise conditions presented statistically significant cortico-acoustic coupling at any of the two frequencies of interest (Fig. 3).

To specifically test our hypothesis of a stronger involvement of the motor cortex in the tracking of natural fast as compared to timecompressed speech, we computed direct contrasts between speech conditions at the frequency matching the mean fast syllable rate $(\sim 8.75 \mathrm{~Hz})$. More specifically, we focused on a putative right articulatory area which we defined as the region of the right motor cortex (BA 4) that showed a cortico-acoustic coherence peak at $\sim 8.75 \mathrm{~Hz}$ (Fig. 3B) and based on previous neuroimaging reports on the involvement of the articulatory cortex in speech perception and production (Brown et al., 2009; Glanz Iljina et al., 2018). Fig. 4A shows the maps of cortico-acoustic coherence for the three computed contrasts. Remarkably, the right articulatory motor cortex showed stronger entrainment to natural fast rate speech, compared to both time-compressed and natural normal speech (Fig. 4B, articulatory ROI). Note that contrasts between speech conditions using the whole set of predefined ROIs (as in Fig. 1) showed an increase of coherence for natural fast as compared to time-compressed speech in the same region as well as in the right auditory and temporal cortex (uncorrected results; see Supplementary Fig. 3). For completeness, we also computed direct pairwise contrasts between speech conditions at $\sim 6.25 \mathrm{~Hz}$ (normal syllable rate range). Results showed significantly stronger coupling to natural speech, both at normal and fast rates, compared to timecompressed speech in the right precentral ROI (see Supplementary Fig. $4)$. The two naturally-produced conditions did not significantly differ from each other at the frequency of normal speech $(\sim 6.25 \mathrm{~Hz})$.

Next, in a supplementary follow-up analysis, we sought to assess differences in cortico-cortical network dynamics associated with processing naturally and artificially accelerated speech. To this end, we measured seed-based phase interaction patterns at $\sim 8.75 \mathrm{~Hz}$ using weighted Phase Lag Index (wPLI). In light of the cortico-acoustic coherence results, we chose to focus the inter-areal coupling analyses on two key ROIs: the right articulatory motor and right auditory areas, as both showed stronger entrainment to natural fast than to time-compressed speech (Figs. 3B, 4 and S3). As illustrated in Supplementary Fig. 5B, the right articulatory motor cortex showed higher inter-areal phase coupling for natural fast than for time-compressed sentences, mainly with the right auditory cortex (BA 41/42) and a left-lateralized network encompassing the inferior parietal cortex (supramarginal and angular gyri BA 39/40), Broca's area (BA 44/45), the primary motor and premotor cortices (BAs 4/6) extending to the supplementary motor area (SMA), the primary sensory cortex (BA 1) and the dorsolateral prefrontal cortex (BA 9/10/46). Direct comparison between natural fast and normal rate speech at the same frequency $(\sim 8.75 \mathrm{~Hz})$ showed enhanced coupling between the right motor cortex and the left lateral premotor cortex (BA 6 including SMA) and left dorsolateral prefrontal cortex (BA 46). 
A

Natural Fast Natural Normal

\section{Natural Fast - Time-compressed}

Cortico-acoustic Coherence Contrasts @ $8.75 \pm 1 \mathrm{~Hz}$

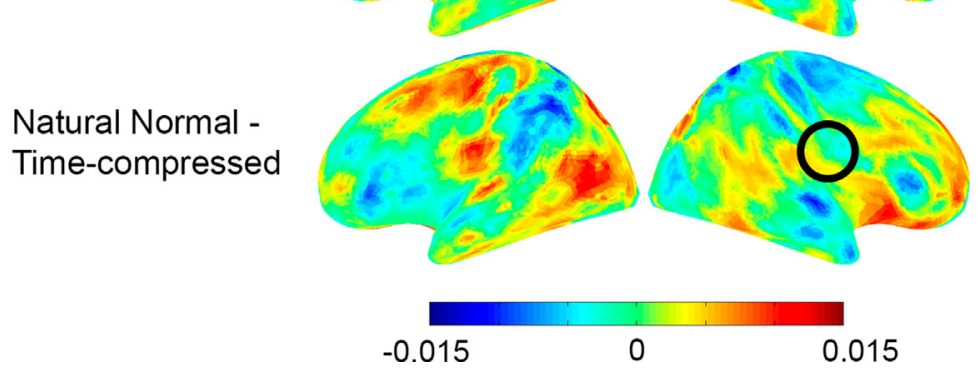

B
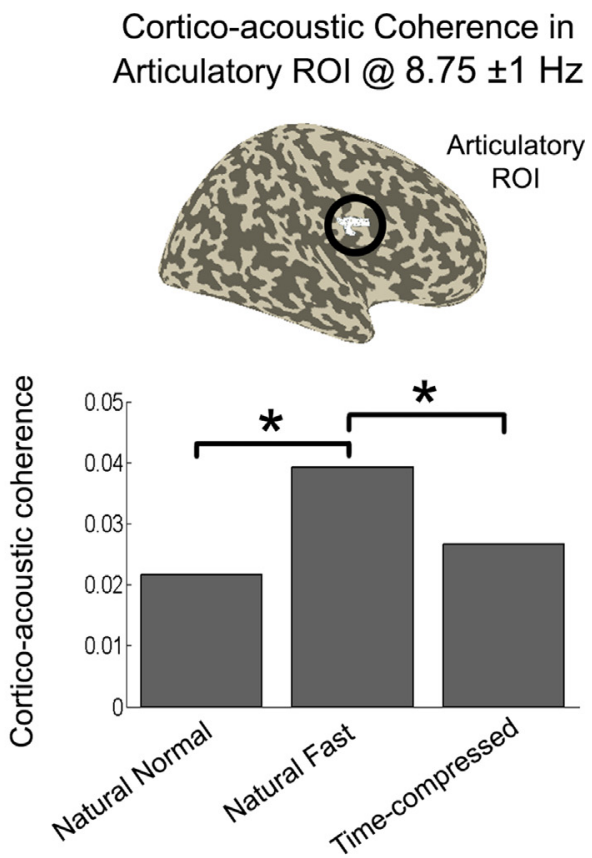

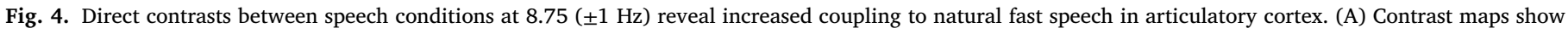

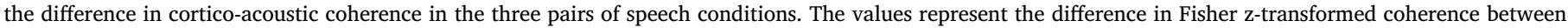

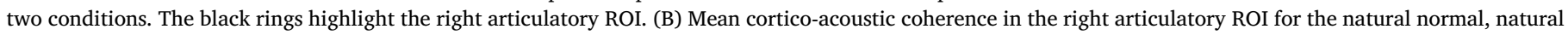
fast and time-compressed conditions. Stars $\left({ }^{*}\right)$ indicate significant differences between conditions ( $t$-test, $\alpha=0.05$, corrected).

When the right auditory cortex was used as the seed (Supplementary Fig. 5A), results showed stronger coupling for the natural fast than for the time-compressed condition mainly with the left inferior parietal cortex (BA 39/40), Broca's area, left inferior part of the primary motor and premotor cortices (BA 4/6), left primary sensory cortex (BA 1) and left primary auditory cortex (BA 41). For the contrast between natural fast and normal rate sentences, the right auditory cortex was more strongly coupled mainly to the bilateral primary motor and premotor cortices (BAs $4 / 6$, more ventrally in the right hemisphere) and right inferior frontal gyrus (BAs 44/45).

Finally, we sought to rule out that the observed increases in coupling between cortical oscillations and the speech envelope can be linked to increases in cortical power at the coupling frequency. To this end, we analyzed source power modulations, contrasting power for the speech encoding period with power measured during a pre-stimulus baseline. The analyses showed significant increases of spectral power at $\sim 6.25 \mathrm{~Hz}$ in the left prefrontal and inferior frontal cortex for all sentence conditions (Supplementary Fig. 6). These areas did not overlap with the regions that exhibited significant cortico-acoustic coherence at this frequency. This was accompanied by significant desynchronization at $\sim 8.75 \mathrm{~Hz}$ in the right temporal cortex in all conditions (except for noise modulated with the envelope of fast rate sentences) and in the left inferior frontal cortex for the two naturally-produced speech conditions (Supplementary Fig. 7). These power reductions in the same areas and frequencies in which we report entrainment to the natural fast stimuli suggest that the observed coupling cannot be attributed to local increases in power.

\section{Discussion}

To date, most of the evidence for brain alignment to speech rate variations has come from research using artificially accelerated speech and has thereby left the issue of natural speech rate changes largely unaddressed. The present MEG study is the first to directly compare brain-tospeech coupling between naturally-produced fast speech and artificially compressed speech. We first showed that neural oscillations in auditory and (pre)motor cortex track natural variations of syllable rate at fre- quencies that specifically reflect the temporal structure of the speech material. Cortical rhythms indeed shift up their coupling frequency to match the faster modulations in natural fast speech. Surprisingly, we did not observe any significant cortical coupling at the same frequency $(\sim 8.75 \mathrm{~Hz})$ when the speech was generated through time-compression. Crucially, direct contrasts between conditions at this higher frequency revealed stronger tracking of speech envelope in the right motor cortex for natural fast than for artificially accelerated speech, possibly reflecting specific mapping to articulatory features of naturally-produced material. Furthermore, although the results of seed-based source-space wPLI analyses did not survive correction for multiple comparison across 8693 sources, the uncorrected statistics suggest that the right articulatory/motor ROI showed enhanced phase synchronization with the left temporo-parietal and motor cortices in the natural fast compared to the time-compressed condition. Finally, we found that the reported corticoacoustic coupling is sensitive to the presence of spectral information in the stimuli and does not only depend on the rhythmic acoustic structure, as no significant increase of coherence is seen for amplitude-modulated noise, despite being generated using the speech signal envelopes.

Brain coupling to the amplitude envelope of naturally-produced speech was found in auditory and precentral regions of the right hemisphere, in agreement with oscillatory-based models of speech perception (Ghitza, 2011; Poeppel, 2003) and previous work showing rightlateralized brain responses to speech envelope (Abrams et al., 2008; Alexandrou et al., 2017; Gross et al., 2013; Luo and Poeppel, 2012). Specific MEG responses in the right pre/postcentral gyri were also recently reported for sequences of random syllables at $4 \mathrm{~Hz}$ (Sheng et al., 2019). Most importantly, our coherence measures revealed that neural oscillatory activity is tuned to speech rate variations: cortical tracking of naturally-produced sentences was observed at frequencies coinciding with the syllable rate of the stimuli. For normal rate speech, cortico-acoustic coherence in auditory and (pre)motor regions increased at $\sim 6.25 \mathrm{~Hz}$, whereas when participants listened to natural fast speech, the peak of coupling shifted up to $\sim 8.75 \mathrm{~Hz}$ (Fig. 3). Note that a significant increase of coherence in the right precentral cortex was also observed for naturally accelerated speech at the lower frequency. Sylla- 
ble frequencies in natural speech tend to overlap between speech rates (Alexandrou et al., 2016). Along this line, the envelope of our natural fast sentences also contains slower frequency components, which may account for the observed pattern of results. In fact, this explanation is consistent with the spectral power density plots (Supplementary Fig. 1). The lack of coupling for normal rate speech at the higher frequency $(\sim 8.75 \mathrm{~Hz})$, which was expected, however underlines the specificity of the reported effects. Similar patterns of brain-to-speech coupling were observed when we contrasted coherence between speech and MEG brain activity with coherence computed for baseline trials (Supplementary Fig. 2). This replication using two distinct methods supports the reliability of our observations.

The neurocognitive mechanism underlying brain-to-speech coupling is still debated (Ding and Simon, 2014; Zoefel, 2018; Zoefel et al., 2018). The dominant model (Ghitza, 2012; Giraud and Poeppel, 2012; Peelle and Davis, 2012) suggests that endogenous neural oscillations actively synchronize (or entrain) to speech envelope. Increasing evidence from EEG and MEG supports this view, in part by showing sustained oscillations after speech stimuli offset (Ding et al., 2016; Doelling et al., 2019; Kayser et al., 2015; Kösem et al., 2018a; Makov et al., 2017; van Bree et al., 2021; Zoefel et al., 2020; Zoefel and VanRullen, 2015). Alternatively, brain-to-speech coupling has been suggested to reflect evoked responses that are elicited by rhythmic acoustic cues in the incoming stimuli, without involving endogenous oscillations (Doelling et al., 2014; Howard and Poeppel, 2010; Kojima et al., 2021; Novembre and Iannetti, 2020; Oganian and Chang, 2019). One prediction of this latter view is that regular repetition of evoked responses should lead to an increase of power at the stimulus frequency. Crucially, in our study, the significant increases of cortico-acoustic coherence for natural normal and fast rate speech were not accompanied by power increases (but rather power suppression at $\sim 8.75 \mathrm{~Hz}$ ) in the same cortical regions and frequencies (Supplementary Figs. 6 and 7). These findings may thus endorse the oscillatory entrainment model and suggest a genuine synchronization phenomenon that cannot be attributed to increases in signal amplitude (see also Kayser et al., 2015; Luo et al., 2013; ten Oever et al., 2017; Zoefel et al., 2018). In addition, one could argue that the lack of a significant increase in cortico-acoustic coherence in the amplitude-modulated noise condition (Fig. 3) also speaks against the evoked response model.

Our results of power increase at $\sim 6.25 \mathrm{~Hz}$ ( $\sim$ theta) to sentences in left prefrontal and inferior frontal cortex may be related to increased working memory load and lexico-semantic retrieval during sentence processing (Bastiaansen et al., 2005; Lam et al., 2016). Besides, the $\sim 8.75 \mathrm{~Hz}$ desynchronization in right anterior temporal and left inferior frontal regions agrees with studies showing alpha band (8-13 Hz) desynchronization during auditory stimulus processing. This is classically thought to reflect enhanced mental operations and thus more active cognitive processing of the signal (Obleser and Weisz, 2012; Weisz et al., 2011). Alpha suppression associated with theta power enhancement in frontal regions have also been reported in response to speech for lexico-semantic processing (Obleser and Weisz, 2012; Shahin et al., 2009).

Our findings first add new evidence to previous work on artificially accelerated speech (Lizarazu et al., 2019; Pefkou et al., 2017) by revealing that cortical oscillations align to envelope modulations at a higher frequency to match the faster syllable rate of naturally-produced speech, despite increased articulatory variation (as compared to timecompressed speech). Such auditory and motor coupling at $\sim 8.75 \mathrm{~Hz}$ fits with results showing three peaks of resting-state theta-band activity in auditory cortex (4.5, 6.5 and $8.5 \mathrm{~Hz})$ as well as intrinsic alpha-band activity (7-13 Hz) in the right precentral gyrus (Keitel and Gross, 2016). It is also of note that the shift in coupling frequency was found for single, relatively short sentences with a syllable rate up to 10 syllables/s (mean $=9.15$ syllables/s). Ahissar et al. (2001) suggested that coupling to short sentences compressed to ratios of $0.35(\sim 9 \mathrm{~Hz})$ and $0.2(\sim 14 \mathrm{~Hz})$ failed in their experiment because neural oscillations may not have had enough time to change their coupling frequency to match that of the stimuli. Although this is a plausible explanation, the present data using naturally-produced material show that even with single and relatively short sentences, neural oscillations are able to adjust their coupling frequency to higher syllable rates.

In line with the study by Keitel et al. (2018), our results emphasize the relevance of assessing neural tracking of speech at frequencies given by stimulus properties rather than in generic frequency bands which may not capture the specific underlying processes at stake. The MEG study by Alexandrou et al. (2018) showed alignment of auditory and parietal cortex oscillations to spontaneously-produced connected speech at slow ( $\sim 2.6$ syllables/s), normal ( $\sim 4.7$ syllables/s) and fast ( $\sim 6.8$ syllables/s) syllable production frequencies (yet slower than our fast rate condition). Despite providing valuable evidence regarding natural speech perception, the authors however examined coupling in the canonical delta $(2-4 \mathrm{~Hz})$ and theta $(4-7 \mathrm{~Hz})$ bands and did not look at potential variations in brain coupling frequency according to speech rates. Although our study used single sentences, we bring novel evidence for cortical alignment to natural syllable rates up to an average of $9 \mathrm{~Hz}$ and at frequencies that are specific to the speech material. Future work should certainly investigate brain coupling to longer extracts of naturally-produced speech at such normal and fast rates and in stimulusbased frequency bins as we did in our study.

Assaneoet al. (2019) reported cortico-acoustic coupling in inferior and middle frontal gyri at $4.5 \mathrm{~Hz}$, as well as enhanced phase coupling between both left and right auditory and motor regions (Assaneo and Poeppel, 2018), when participants listened to synthesized syllables at the same rate (see also Keitel et al., 2018 for delta motor coupling to normal rate sentences embedded in noise). Sheng et al. (2019) also found specific tracking of syllables at a rate of $4 \mathrm{~Hz}$ in the right pre/postcentral cortex. Here, we show that right (pre)motor regions synchronize their oscillatory activity to more complex speech stimuli (i.e. meaningful sentences) that are naturally produced at faster rates (up to 9.15 syllables/s on average). Similar to auditory cortex, oscillations in the motor cortex are therefore able to shift up their coupling frequency to follow the natural increase in syllable rate. Remarkably, analyses at $\sim 8.75 \mathrm{~Hz}$ revealed tracking of natural fast speech in a region of the ventral motor cortex (BA 4) which coordinates are very close to those of the articulatory cortex (mouth motor region) identified in neuroimaging studies on speech production and/or perception (Brown et al., 2009; Glanz Iljina et al., 2018; Pulvermuller et al., 2006). Coupling to naturally-produced fast speech in this ventral motor region may therefore reflect articulatory mechanisms and more particularly simulation of the syllable production rhythm of heard sentences.

Motor regions have also been suggested to contribute to top-down auditory processing and to the establishment of auditory temporal predictions (Park et al., 2015; Keitel et al., 2017; Morillon and Baillet, 2017; Rimmele et al., 2018). Our findings, along with those of few other studies (Alexandrou et al., 2018; Assaneo et al., 2019; Sheng et al., 2019), underline that motor regions do not only exert a modulatory control but directly track the speech signal. Neural oscillations in motor regions align to low-frequency modulations in natural speech, both at normal and fast rates, possibly reflecting sensorimotor integration processes. In the present study, participants listened to sentences with the same syntactic structure, it is therefore possible that motor regions tracked and synchronized to syllable rate regularities so as to predict the occurrence of the next syllables. Such a predictive timing mechanism may facilitate the syllabic parsing of the unfolding speech stream by the auditory cortex (Morillon and Baillet, 2017).

No significant cortical coupling to time-compressed sentences was observed at the corresponding frequency $(\sim 8.75 \mathrm{~Hz})$, which is at odds with previous work, at least regarding auditory cortex oscillations (Ahissar et al., 2001; Hertrich et al., 2012; Pefkou et al., 2017). To the best of our knowledge, these studies have mostly focused on neural coupling in the auditory cortex and we are only aware of a few studies that documented theta (4-7 Hz) synchronization to degraded (though noisevocoded, not time-compressed) speech in distributed cortical networks 
including the motor cortex (Millman et al., 2015; Peelle et al., 2013). Note from Fig. 3B that coherence maps tend to show increase of coherence, although weaker, for time-compressed speech at $\sim 8.75 \mathrm{~Hz}$ in a similar fronto-temporal network as for natural fast rate speech, however this did not survive statistical correction despite a sample size of 23 analyzed participants. Some methodological considerations may account, at least partly, for this apparent discrepancy between studies. Unlike previous work, we mixed two types of accelerated speech, with sentences pseudorandomly presented to participants. This may have elicited different coupling effects because of the attention-demanding nature of natural fast speech, which may be more difficult to process than artificially manipulated speech. In line with previous observations (e.g., Guiraud et al., 2018; Janse, 2004), our online intelligibility experiment confirmed that naturally accelerated sentences were slightly more difficult to comprehend than time-compressed sentences. Yet they were associated with stronger cortico-acoustic coupling at $\sim 8.75 \mathrm{~Hz}$ in auditory and motor regions. In fact, this stronger neural coupling to sentences in the natural fast compared to the time-compressed conditions was also visible on the direct contrast map at the same frequency $(\sim 8.75 \mathrm{~Hz}$; see Supplementary Fig. 3). This suggests that the strength of neural entrainment may not be correlated with the degree of intelligibility but instead may be driven by the specific spectro-temporal signature of natural speech. Evidence that intelligibility depends on the strength of neural tracking of speech remains contradictory (Baltzell et al., 2017; Doelling et al., 2014; Gross et al., 2013; Howard and Poeppel, 2010; Millman et al., 2015; Peelle et al., 2013; Zoefel and VanRullen, 2016). Enhanced coupling to intelligible vs unintelligible speech has been reported using vocoded or time-reversed sentences (Gross et al., 2013; Peelle et al., 2013; see also Zoefel and VanRullen, 2015). While these manipulations degrade speech intelligibility, they arguably also distort other properties of the stimuli that can affect the quality of cortico-acoustic entrainment. For instance, reversed speech alters the spectro-temporal properties of the signal, with slow onsets and abrupt offsets, which affects the phonotactic structure and leads to implausible articulatory patterns (Narain et al., 2003; Pellegrino et al., 2010). This makes it difficult to decide whether the reduced entrainment for reversed speech is related to the drop of intelligibility or if it is actually caused by acoustic-phonetic changes that result from the speech distortion (see also Baltzell et al., 2017). Our findings of stronger coupling to natural fast (although less intelligible) speech, compared to time-compressed (more intelligible) speech provides new insights into this debate. Our data suggest that stronger entrainment is not necessarily associated with better intelligibility. The cortico-acoustic coupling patterns revealed here seem to be rather associated with the encoding of natural high-level spectrotemporal features (including phonetic information). Interestingly, the stronger cortico-acoustic coupling was in particular found in the right superior temporal gyrus (Fig. 3B), in line with the role of this region in spectral processing (Obleser et al., 2008; Zatorre and Belin, 2001).

Importantly, the way speech rate was sped up in our study may have affected brain oscillatory responses to sentences. Uttering speech at a fast rate is a nonlinear phenomenon whereby segments are not reduced similarly, partly because of articulatory constraints, thus enhancing the prosodic pattern (Janse, 2004). By contrast, artificially accelerated speech was obtained by linear compression, meaning that all segments were shortened in the same way. This leads to unnatural patterns that are not biologically (articulatory-speaking) plausible and may thus not resonate in brain motor regions (or less so) as naturallyaccelerated speech does. Whereas we are indeed rather accustomed to and can reproduce natural fast sentences relatively easily, this is not the case for linearly time-compressed speech. Crucially, our results revealed significant coupling of the right motor cortex at $\sim 8.75 \mathrm{~Hz}$ to naturally accelerated but not to time-compressed speech, despite having the same syllable rate. Direct contrasts between the two conditions corroborated this result (Fig. 4). This major finding may reflect differences in the structure of the two types of signals and demonstrate specific mapping, in the motor cortex, to articulatory features that char- acterize naturally-produced fast speech as compared to synthesized fast speech.

Remarkably, our contrasts analyses at the lower frequency $(\sim 6.25 \mathrm{~Hz})$ highlighted significantly stronger tuning in the precentral cortex for naturally-produced speech, either at a normal or fast rate, than for time-compressed speech (see Supplementary Fig. 4). No difference was observed when we contrasted the natural normal and fast rate conditions. This finding is particularly interesting and can be interpreted in the framework of studies showing that the motor cortex intrinsically oscillates in the theta band (Giraud et al., 2007; Keitel and Gross, 2016; Poeppel and Assaneo, 2020). Given that our $\sim 6.25 \mathrm{~Hz}$ frequency of interest fell within this range, our results may emphasize the tendency of the motor cortex to preferentially align to natural rather than to artificially speech. Hence, the motor cortex more strongly resonates, at its own preferred rhythm, with speech perception when the signal is naturally produced, irrespective of the syllable rate, than when it has been artificially manipulated. This may indicate enhanced synchronization to articulatory patterns that are most prominent in natural vs compressed speech as well as increased sensorimotor integration required to match phonological and articulatory templates of natural speech.

Supplementary cortico-cortical coupling analyses at $\sim 8.75 \mathrm{~Hz}$ revealed enhanced connectivity between the right motor/articulatory cortex and the left inferior parietal and (pre)motor cortices and Broca's area during perception of natural fast with respect to time-compressed sentences (Supplementary Fig. 5). We also found the right auditory cortex to be more strongly coupled to these same regions in the naturally accelerated condition. This left-lateralized temporo-parieto-frontal network is part of the sensorimotor dorsal stream thought to instantiate forward and inverse articulatory-orosensory-auditory internal models to facilitate speech perception, especially under challenging conditions (Callan et al., 2004; Hickok et al., 2011; Hickok and Poeppel, 2007; Lima et al., 2016; Nuttall et al., 2016; Rauschecker and Scott, 2009). In line with this, we show that listening to naturally-produced fast speech specifically increases the functional connectivity between the right motor cortex, which synchronizes to syllable rate, and regions of the left dorsal stream (extending to the SMA). Interestingly, such interhemispheric coupling was also described during the perception of distorted speech in a recent dual-coil TMS study (Nuttall et al., 2018). The results showed that disrupting the right ventral premotor cortex inhibited left motor cortex excitability, as reflected by decreased motor evoked potentials over lip muscles, when participants listened to imprecisely articulated syllables as compared to clear speech.

Consistent with the involvement of reverberant, dynamic bilateral speech motor networks in speech perception (Skipper et al., 2017), our findings thus show that the right motor cortex specifically entrains to the syllable rate of naturally-accelerated speech, and is more strongly coupled to left parietal and (pre)motor regions when perceiving natural fast as compared to time-compressed speech. This may reflect enhanced resonance to the increased articulatory complexity of natural fast speech, which may more strongly rely on internal models than artificially accelerated speech to efficiently map distorted perceptually representations to stored orosensory and articulatory representations. Of course, one should keep in mind that the cortico-cortical phase synchronization patterns observed in the present study (Supplementary Fig. 5) need to be interpreted with caution as the effects did not survive correction for multiple comparisons across the 8693 sources.

Previous work documented auditory cortex alignment to both verbal and non-verbal stimuli (Doelling et al., 2014; Howard and Poeppel, 2010; Luo and Poeppel, 2012; Molinaro and Lizarazu, 2017; Rimmele et al., 2015; Teng et al., 2018). Our data show that amplitudemodulated noise did not significantly "entrain" cortical oscillations at the two frequencies of interest, although these stimuli were generated using the temporal envelopes of normal rate and fast rate sentences. In other words, increased cortico-acoustic coherence was found for normal rate and fast rate sentences but not for stimuli with the same envelope characteristics but which lack fine spectro-temporal information 
found in speech. A possible interpretation of this result is that brain coupling to natural syllable rate variations does not only reflect passive tracking of the rhythmic acoustic structure present in the slow modulations of the amplitude envelope, but that it is sensitive to higher-level features carried by spectral modulations, in line with previous studies (Ding et al., 2014; Peelle et al., 2013; Zoefel and VanRullen, 2015). An alternative explanation is that stronger coupling to sentences than to amplitude-modulated noise could result from top-down influence of lexico-semantic information (given the rich semantic content of our sentence material). Our results cannot currently disentangle these two interpretations and future work comparing brain oscillatory responses to intelligible naturally-produced speech to those induced by unintelligible speech with the same spectro-temporal complexity (Millman et al., 2015; Molinaro and Lizarazu, 2017; Peelle et al., 2013) as well as to linguistic stimuli without any semantic content such as foreign language are certainly needed.

\section{Conclusions}

Our findings shed new light onto the brain oscillatory dynamics that mediate natural speech perception, by revealing that neural oscillations are tuned to natural speech rate variations at frequencies that match the syllabic structure of the acoustic input. We found such frequencyspecific coupling not only in auditory but also in (pre)motor regions, emphasizing their role in speech sensorimotor integration. Our results also provide unprecedented evidence for a stronger oscillatory coupling in right motor cortex to naturally accelerated compared to artificially manipulated speech. In addition, our follow-up cortico-cortical connectivity analysis suggests enhanced coupling between right motor cortex and left parietal and motor regions for natural fast speech. These observations likely reflect enhanced distributed tracking and encoding of articulatory features of naturally accelerated speech. Our data thus highlight the relevance of using both natural speech material (despite being more methodologically constraining) and stimulus-specific (vs generic) frequencies to thoroughly assess brain-to-speech alignment in future studies. The prominent role of right auditory but also motor areas unveiled by our study might provide valuable insights for the advancement of oscillatory models of speech perception and production. Finally, the proposed paradigm may also prove of high interest to investigate the developmental trajectory of neural tracking of speech, both in children with typical and atypical language development.

\section{Credit authorship contribution statement}

Ana Sofía Hincapié Casas: Software, Formal analysis, Visualization, Writing - original draft. Tarek Lajnef: Software, Formal analysis. Annalisa Pascarella: Software, Formal analysis, Visualization. Hélène Guiraud-Vinatea: Conceptualization, Methodology. Hannu Laaksonen: Software. Dimitri Bayle: Software. Karim Jerbi: Conceptualization, Methodology, Visualization, Funding acquisition, Supervision, Project administration, Writing - original draft, Writing - review \& editing. Véronique Boulenger: Conceptualization, Methodology, Investigation, Resources, Funding acquisition, Supervision, Project administration, Writing - original draft, Writing - review \& editing.

\section{Acknowledgments}

We would like to thank two Reviewers for their insightful comments on a previous version of the manuscript, Damien Gouy for recording the sentences, Emmanuel Ferragne for his help with Praat software, and Mélanie Canault and François Pellegrino for discussions on articulatory phonetics and signal processing respectively. We are also grateful to Sébastien Daligault and Claude Delpuech from the CERMEP-Imagerie du Vivant of Lyon for assisting in MEG data acquisition, to Mainak Jas for his implementation of the algorithm for automatic parameterization of neural power spectral densities (PSDs) and to Sébastien Flavier for implementing the online behavioral intelligibility experiment.

\section{Funding sources}

V.B. was supported by funding from the French National Research Agency (ANR) for the ODYSSEE project (ANR-11-JSH2 0051 ) and by the Laboratory of Excellence (LabEx) ASLAN (ANR-10-LABX-0081) of Université de Lyon within the program "Investissements d'Avenir" (ANR11-IDEX-0007) of the French government operated by the ANR. H.G. was funded by a doctoral scholarship from the LabEx ASLAN (ANR10-LABX-0081). H.L. was supported by a post-doctoral grant from the ANR for the ODYSSEE project (ANR-11-JSH2 005 1). K.J. was supported by funding from the Canada Research Chairs program and a Discovery Grant (RGPIN-2015-04854) from the Natural Sciences and Engineering Research Council of Canada, a New Investigators Award from the Fonds de Recherche du Québec - Nature et Technologies (2018-NC-206005) and an IVADO-Apogée fundamental research project grant.

\section{Data availability statement}

The data that support the findings of this study, as well as the codes used for analysis, are available from the corresponding author upon reasonable request. Some data of the project are indeed currently still under analysis. During this period, data sharing will therefore depend on the aim of the request. The source of the data and the authors' names of the present manuscript will need to be cited.

\section{Supplementary materials}

Supplementary material associated with this article can be found, in the online version, at doi:10.1016/j.neuroimage.2021.118577.

\section{References}

Abrams, D.A., Nicol, T., Zecker, S., Kraus, N., 2008. Right-hemisphere auditory cortex is dominant for coding syllable patterns in speech. J. Neurosci. 28 (15), 3958-3965. doi:10.1523/JNEUROSCI.0187-08.2008.

Adank, P., Janse, E., 2009. Perceptual learning of time-compressed and natural fast speech. J. Acoust. Soc. Am. 126 (5), 2649. doi:10.1121/1.3216914.

Ahissar, E., Nagarajan, S., Ahissar, M., Protopapas, A., Mahncke, H., Merzenich, M.M., 2001. Speech comprehension is correlated with temporal response patterns recorded from auditory cortex. Proc. Natl. Acad. Sci. 98 (23), 13367-13372. doi:10.1073/pnas.201400998.

Alexandrou, A.M., Saarinen, T., Kujala, J., Salmelin, R., 2016. A multimodal spectral approach to characterize rhythm in natural speech. J. Acoust. Soc. Am. 139 (1), 215226. doi:10.1121/1.4939496.

Alexandrou, A.M., Saarinen, T., Kujala, J., Salmelin, R., 2018. Cortical tracking of global and local variations of speech rhythm during connected natural speech perception. J. Cogn. Neurosci. 30 (11), 1704-1719. doi:10.1162/jocn_a_01295.

Alexandrou, A.M., Saarinen, T., Mäkelä, S., Kujala, J., Salmelin, R., 2017. The right hemisphere is highlighted in connected natural speech production and perception. Neuroimage 152, 628-638. doi:10.1016/j.neuroimage.2017.03.006.

Assaneo, M.F., Poeppel, D., 2018. The coupling between auditory and motor cortices is rate-restricted: evidence for an intrinsic speech-motor rhythm. Sci. Adv. 4 (2). doi:10.1126/sciadv.aao3842.

Assaneo, M.F., Ripolles, P., Orpella, J., Lin, W.M., de Diego-Balaguer, R., Poeppel, D. 2019. Spontaneous synchronization to speech reveals neural mechanisms facilitating language learning. Nat. Neurosci. 22 (4), 627-632. doi:10.1038/s41593-019-0353-z.

Baltzell, L.S., Srinivasan, R., Richards, V.M., 2017. The effect of prior knowledge and intelligibility on the cortical entrainment response to speech. J. Neurophysiol. 118 (6), 3144-3151. doi:10.1152/jn.00023.2017.

Bastiaansen, M., Linden, M.van der, Keurs, M.ter, Dijkstra, T., Hagoort, P., 2005. Theta responses are involved in lexical-semantic retrieval during language processing. J. Cogn. Neurosci. 17 (3), 530-541. doi:10.1162/0898929053279469.

Berry, J.J., 2011. Speaking rate effects on normal aspects of articulation: outcomes and issues. Perspect. Speech Sci. Orofac. Disord. 21 (1), 15-26. doi:10.1044/ssod21.1.15.

Boersma, P., \& Weenik, D. (2012). Doing phonetics by computer [Computer program]. http://www.praat.org/

Bosker, H.R., Ghitza, O., 2018. Entrained theta oscillations guide perception of subsequent speech: behavioral evidence from rate normalisation. Lang. Cogn. Neurosci. 33 (8), 955-967. doi:10.1080/23273798.2018.1439179.

Brown, S., Laird, A.R., Pfordresher, P.Q., Thelen, S.M., Turkeltaub, P., Liotti, M., 2009. The somatotopy of speech: phonation and articulation in the human motor cortex. Brain Cogn. 70 (1), 31-41. doi:10.1016/j.bandc.2008.12.006. 
Callan, D.E., Jones, J.A., Callan, A.M., Akahane-Yamada, R., 2004. Phonetic perceptual identification by native- and second-language speakers differentially activates brain regions involved with acoustic phonetic processing and those involved with articulatory-auditory/orosensory internal models. Neuroimage 22 (3), 1182-1194. doi:10.1016/j.neuroimage.2004.03.006.

Chandrasekaran, C., Trubanova, A., Stillittano, S., Caplier, A., Ghazanfar, A.A., 2009. The natural statistics of audiovisual speech. PLoS Comput. Biol. 5 (7), e1000436. doi:10.1371/journal.pcbi.1000436.

Ding, N., Chatterjee, M., Simon, J.Z., 2014. Robust cortical entrainment to the speech envelope relies on the spectro-temporal fine structure. Neuroimage 88, 41-46. doi:10.1016/j.neuroimage.2013.10.054.

Ding, N., Melloni, L., Zhang, H., Tian, X., Poeppel, D., 2016. Cortical tracking of hierarchical linguistic structures in connected speech. Nat. Neurosci. 19 (1), 158-164. doi:10.1038/nn.4186

Ding, N., \& Simon, J.Z. (2014). Cortical entrainment to continuous speech: functional roles and interpretations. Front. Human Neurosci., 8. 10.3389/fnhum.2014.00311

Doelling, K.B., Arnal, L.H., Ghitza, O., Poeppel, D., 2014. Acoustic landmarks drive deltatheta oscillations to enable speech comprehension by facilitating perceptual parsing. Neuroimage 85, 761-768. doi:10.1016/j.neuroimage.2013.06.035.

Doelling, K.B., Assaneo, M.F., Bevilacqua, D., Pesaran, B., Poeppel, D., 2019. An oscillator model better predicts cortical entrainment to music. Proc. Natl. Acad. Sci. U.S.A. 116 (20), 10113-10121. doi:10.1073/pnas.1816414116, Scopus.

Evans, S., Davis, M.H., 2015. Hierarchical organization of auditory and motor representations in speech perception: evidence from searchlight similarity analysis. Cerebral Cortex (New York, NY) 25 (12), 4772-4788. doi:10.1093/cercor/bhv136.

Ferragne, E., Flavier, S., \& Fressard, C. (2012). Recording of Oral Corpora Made Easy (ROCme): logiciel d'enregistrement de corpus.

Ghitza, O., 2011. Linking speech perception and neurophysiology: speech decoding guided by cascaded oscillators locked to the input rhythm. Front. Psychol. 2. doi:10.3389/fpsyg.2011.00130.

Ghitza, O., 2012. On the role of theta-driven syllabic parsing in decoding speech: intelligibility of speech with a manipulated modulation spectrum. Front. Psychol. 3. doi:10.3389/fpsyg.2012.00238.

Giraud, A.-.L., Kleinschmidt, A., Poeppel, D., Lund, T.E., Frackowiak, R.S.J., Laufs, H., 2007. Endogenous cortical rhythms determine cerebral specialization for speech perception and production. Neuron 56 (6), 1127-1134. doi:10.1016/j.neuron.2007.09.038.

Giraud, A.-.L., Poeppel, D., 2012. Cortical oscillations and speech processing: emerging computational principles and operations. Nat. Neurosci. 15 (4), 511-517. doi: $10.1038 / \mathrm{nn} .3063$.

Glanz Iljina, O., Derix, J., Kaur, R., Schulze-Bonhage, A., Auer, P., Aertsen, A., Ball, T., 2018. Real-life speech production and perception have a shared premotor-cortical substrate. Sci. Rep. 8 (1), 8898. doi:10.1038/s41598-018-26801-X.

Gross, J., Hoogenboom, N., Thut, G., Schyns, P., Panzeri, S., Belin, P., Garrod, S., 2013. Speech rhythms and multiplexed oscillatory sensory coding in the human brain. PLoS Biol. 11 (12), e1001752. doi:10.1371/journal.pbio.1001752.

Gross, J., Kujala, J., Hämäläinen, M., Timmermann, L., Schnitzler, A., Salmelin, R., 2001. Dynamic imaging of coherent sources: studying neural interactions in the human brain. Proc. Natl. Acad. Sci. U.S.A. 98 (2), 694-699.

Guiraud, H., Bedoin, N., Krifi-Papoz, S., Herbillon, V., Caillot-Bascoul, A., GonzalezMonge, S., Boulenger, V., 2018. Don't speak too fast! Processing of fast rate speech in children with specific language impairment. PLoS ONE 13 (1), e0191808. doi:10.1371/journal.pone.0191808.

Haller, M., Donoghue, T., Peterson, E., Paroma, V., Sebastian, P., Gao, R., Noto, T., Knight, R.T., Shestyuk, A., Voytek, B., 2018. Parameterizing neural power spectra.. bioRxiv. https://www.biorxiv.org/content/10.1101/299859v1.

Hertrich, I., Dietrich, S., Trouvain, J., Moos, A., Ackermann, H., 2012. Magnetic brain activity phase-locked to the envelope, the syllable onsets, and the fundamental frequency of a perceived speech signal. Psychophysiology 49 (3), 322-334. doi:10.1111/j.1469-8986.2011.01314.x

Hickok, G., Houde, J., Rong, F., 2011. Sensorimotor integration in speech processing: computational basis and neural organization. Neuron 69 (3), 407-422. doi:10.1016/j.neuron.2011.01.019.

Hickok, G., Poeppel, D., 2007. The cortical organization of speech processing. Nat. Rev. Neurosci. 8 (5), 393-402. doi:10.1038/nrn2113.

Howard, M.F., Poeppel, D., 2010. Discrimination of speech stimuli based on neuronal response phase patterns depends on acoustics but not comprehension. J. Neurophysiol. 104 (5), 2500-2511. doi:10.1152/jn.00251.2010.

Janse, E., 2004. Word perception in fast speech: artificially time-compressed vs. naturally produced fast speech. Speech Commun. 42 (2), 155-173. doi:10.1016/j.specom.2003.07.001

Kayser, S.J., Ince, R.A.A., Gross, J., Kayser, C., 2015. Irregular speech rate dissociates auditory cortical entrainment, evoked responses, and frontal alpha. J. Neurosci. 35 (44), 14691-14701. doi:10.1523/JNEUROSCI.2243-15.2015.

Keitel, A., Gross, J., 2016. Individual human brain areas can be identified from their characteristic spectral activation fingerprints. PLoS Biol. 14 (6), e1002498. doi:10.1371/journal.pbio.1002498.

Keitel, A., Gross, J., Kayser, C., 2018. Perceptually relevant speech tracking in auditory and motor cortex reflects distinct linguistic features. PLoS Biol. 16 (3), e2004473. doi:10.1371/journal.pbio.2004473.

Keitel, A., Ince, R.A.A., Gross, J., Kayser, C., 2017. Auditory cortical delta-entrainment interacts with oscillatory power in multiple fronto-parietal networks. Neuroimage 147, 32-42. doi:10.1016/j.neuroimage.2016.11.062.

Kojima, K., Oganian, Y., Cai, C., Findlay, A., Chang, E., Nagarajan, S., 2021. Lowfrequency neural tracking of speech amplitude envelope reflects the convolu- tion of evoked responses to acoustic edges, not oscillatory entrainment. BioRxiv doi:10.1101/2020.04.02.022616, 2020.04.02.022616.

Kösem, A., Bosker, H.R., Takashima, A., Meyer, A., Jensen, O., Hagoort, P., 2018a. Neural entrainment determines the words we hear. Current Biol. 28 (18), 2867-2875. doi:10.1016/j.cub.2018.07.023, e3.

Kösem, A., Bosker, H.R., Takashima, A., Meyer, A., Jensen, O., Hagoort, P., 2018b. Neural entrainment determines the words we hear. Current Biol. 28 (18), 2867-2875. doi:10.1016/j.cub.2018.07.023, e3.

Lacadie, C.M., Fulbright, R.K., Constable, R.T., Papademetris, X., 2008. More Accurate talairach coordinates for neuroimaging using nonlinear registration. Neuroimage 42 (2), 717-725. doi:10.1016/j.neuroimage.2008.04.240.

Lam, N.H.L., Schoffelen, J.-.M., Uddén, J., Hultén, A., Hagoort, P., 2016. Neural activity during sentence processing as reflected in theta, alpha, beta, and gamma oscillations. Neuroimage 142, 43-54. doi:10.1016/j.neuroimage.2016.03.007.

Lima, C.F., Krishnan, S., Scott, S.K., 2016. Roles of supplementary motor areas in auditory processing and auditory imagery. Trends Neurosci. 39 (8), 527-542. doi:10.1016/j.tins.2016.06.003.

Lizarazu, M., Lallier, M., Molinaro, N., 2019. Phase-amplitude coupling between theta and gamma oscillations adapts to speech rate. Ann. N. Y. Acad. Sci. 1453 (1), 140152. doi:10.1111/nyas. 14099

Luo, H., Poeppel, D., 2012. Cortical oscillations in auditory perception and speech: evidence for two temporal windows in human auditory cortex. Front. Psychol. 3. doi:10.3389/fpsyg.2012.00170.

Luo, H., Tian, X., Song, K., Zhou, K., Poeppel, D., 2013. Neural response phase tracks how listeners learn new acoustic representations. Current Biol. 23 (11), 968-974. doi:10.1016/j.cub.2013.04.031.

Makov, S., Sharon, O., Ding, N., Ben-Shachar, M., Nir, Y., Golumbic, E.Z., 2017. Sleep disrupts high-level speech parsing despite significant basic auditory processing. J. Neurosci. 37 (32), 7772-7781. doi:10.1523/JNEUROSCI.0168-17.2017.

Maris, E., Oostenveld, R., 2007. Nonparametric statistical testing of EEG- and MEG-data. J. Neurosci. Methods 164 (1), 177-190. doi:10.1016/j.jneumeth.2007.03.024.

Millman, R.E., Johnson, S.R., Prendergast, G., 2015. The role of phase-locking to the temporal envelope of speech in auditory perception and speech intelligibility. J. Cogn. Neurosci. 27 (3), 533-545. doi:10.1162/jocn_a 00719.

Molinaro, N., Lizarazu, M., 2017. Delta(but not theta)-band cortical entrainment involves speech-specific processing. Eur. J. Neurosci. doi:10.1111/ejn.13811, O(0).

Morillon, B., Baillet, S., 2017. Motor origin of temporal predictions in auditory attention. Proc. Natl. Acad. Sci. U.S.A. 114 (42), E8913-E8921. doi:10.1073/pnas.1705373114.

Moulines, E., Charpentier, F., 1990. Pitch-synchronous waveform processing techniques for text-to-speech synthesis using diphones. Speech Commun 9 (5-6), 453-467. doi:10.1016/0167-6393(90)90021-Z

Narain, C., Scott, S.K., Wise, R.J.S., Rosen, S., Leff, A., Iversen, S.D., Matthews, P.M., 2003. Defining a Left-lateralized Response Specific to Intelligible Speech Using fMRI. Cerebral Cortex 13 (12), 1362-1368. doi:10.1093/cercor/bhg083.

Nolte, G., 2003. The magnetic lead field theorem in the quasi-static approximation and its use for magnetoencephalography forward calculation in realistic volume conductors. Phys. Med. Biol. 48 (22), 3637-3652. doi:10.1088/0031-9155/48/22/002.

Novembre, G., Iannetti, G.D., 2020. Hyperscanning alone cannot prove causality. multibrain stimulation can. Trends. Cogn. Sci. (Regul. Ed.) doi:10.1016/j.tics.2020.11.003.

Nuttall, H.E., Kennedy-Higgins, D., Devlin, J.T., Adank, P., 2018. Modulation of intra- and inter-hemispheric connectivity between primary and premotor cortex during speech perception. Brain Lang. 187, 74-82. doi:10.1016/j.bandl.2017.12.002.

Nuttall, H.E., Kennedy-Higgins, D., Hogan, J., Devlin, J.T., Adank, P., 2016. The effect of speech distortion on the excitability of articulatory motor cortex. Neuroimage 128 , 218-226. doi:10.1016/j.neuroimage.2015.12.038.

Obleser, J., Eisner, F., Kotz, S.A., 2008. Bilateral speech comprehension reflects differential sensitivity to spectral and temporal features. J. Neurosci. 28 (32), 8116-8123. doi:10.1523/JNEUROSCI.1290-08.2008.

Obleser, J., Weisz, N., 2012. Suppressed alpha oscillations predict intelligibility of speech and its acoustic details. Cerebral Cortex 22 (11), 2466-2477. doi:10.1093/cercor/bhr325.

Oganian, Y., Chang, E.F., 2019. A speech envelope landmark for syllable encoding in human superior temporal gyrus. Sci. Adv. 5 (11). doi:10.1126/sciadv.aay6279, eaay6279.

Okada, K., Rong, F., Venezia, J., Matchin, W., Hsieh, I.-.H., Saberi, K., Serences, J.T., Hickok, G., 2010. Hierarchical organization of human auditory cortex: evidence from acoustic invariance in the response to intelligible speech. Cerebral Cortex 20 (10), 2486-2495. doi:10.1093/cercor/bhp318.

Oldfield, R.C., 1971. The assessment and analysis of handedness: the Edinburgh inventory. Neuropsychologia 9 (1), 97-113. doi:10.1016/0028-3932(71)90067-4.

Oostenveld, R., Fries, P., Maris, E., Schoffelen, J.-.M., 2011. FieldTrip: open source software for advanced analysis of MEG, EEG, and invasive electrophysiological data. Comput. Intell. Neurosci. doi:10.1155/2011/156869.

Osnes, B., Hugdahl, K., Specht, K., 2011. Effective connectivity analysis demonstrates involvement of premotor cortex during speech perception. Neuroimage 54 (3), 24372445. doi:10.1016/j.neuroimage.2010.09.078.

Palva, J.M., Wang, S.H., Palva, S., Zhigalov, A., Monto, S., Brookes, M.J., Schoffelen, J.-.M., Jerbi, K., 2018. Ghost interactions in MEG/EEG source space: a note of caution on inter-areal coupling measures. Neuroimage 173, 632-643. doi:10.1016/j.neuroimage.2018.02.032.

Papademetris, X., Jackowski, M.P., Rajeevan, N., DiStasio, M., Okuda, H., Constable, R.T., Staib, L.H., 2006. BioImage suite: an integrated medical image analysis suite: an update. Insight J. 209.

Park, H., Ince, R.A.A., Schyns, P.G., Thut, G., Gross, J., 2015. Frontal top-down signals increase coupling of auditory low-frequency oscillations to continuous speech in human listeners. Current Biol. 25 (12), 1649-1653. doi:10.1016/j.cub.2015.04.049. 
Peelle, J.E., Davis, M.H., 2012. Neural oscillations carry speech rhythm through to comprehension. Front. Psychol. 3. doi:10.3389/fpsyg.2012.00320.

Peelle, J.E., Gross, J., Davis, M.H., 2013. Phase-locked responses to speech in human auditory cortex are enhanced during comprehension. Cerebral Cortex 23 (6), 1378-1387. doi:10.1093/cercor/bhs118.

Pefkou, M., Arnal, L.H., Fontolan, L., Giraud, A.-.L., 2017. Theta- and beta-band neural activity reflect independent syllable tracking and comprehension of time-compressed speech. J. Neurosci. 2816-2882. doi:10.1523/JNEUROSCI.2882-16.2017.

Pellegrino, F., Coupé, C., Marsico, E., 2011. Across-language perspective on speech information rate. Language (Baltim) 87, 539-558. doi:10.2307/23011654

Pellegrino, F., Ferragne, E., Meunier, F., 2010. 2010, a speech oddity: phonetic transcription of reversed speech. In: Proceedings of the Conference of the International Speech Communication Association (Interpseech), pp. 1221-1224.

Poeppel, D., 2003. The analysis of speech in different temporal integration windows: cerebral lateralization as 'asymmetric sampling in time. Speech Commun 41 (1), 245-255. doi:10.1016/S0167-6393(02)00107-3.

Poeppel, D., Assaneo, M.F., 2020. Speech rhythms and their neural foundations. Nature Rev. Neurosci. 21 (6), 322-334. doi:10.1038/s41583-020-0304-4.

Pulvermüller, F., Fadiga, L., 2010. Active perception: sensorimotor circuits as a cortical basis for language. Nature Rev. Neurosci. 11 (5), 351-360. doi:10.1038/nrn2811.

Pulvermuller, F., Huss, M., Kherif, F., Moscoso del Prado Martin, F., Hauk, O., Shtyrov, Y., 2006. Motor cortex maps articulatory features of speech sounds. Proc. Natl. Acad. Sci. 103 (20), 7865-7870. doi:10.1073/pnas.0509989103.

Rauschecker, J.P., Scott, S.K., 2009. Maps and streams in the auditory cortex: nonhuman primates illuminate human speech processing. Nat. Neurosci. 12 (6), 718-724. doi:10.1038/nn.2331.

Riecke, L., Formisano, E., Sorger, B., Başkent, D., Gaudrain, E., 2018. Neural entrainment to speech modulates speech intelligibility. Current Biol. 28 (2), 161-169. doi:10.1016/j.cub.2017.11.033, e5.

Rimmele, J.M., Golumbic, E.Z., Schröger, E., Poeppel, D., 2015. The effects of selective attention and speech acoustics on neural speech-tracking in a multi-talker scene. Cortex J. Devoted Study Nervous Sys.Behav. 68, 144-154. doi:10.1016/j.cortex.2014.12.014.

Rimmele, J.M., Morillon, B., Poeppel, D., Arnal, L.H., 2018. Proactive sensing of periodic and aperiodic auditory patterns. Trends Cogn. Sci. (Regul. Ed.) 22 (10), 870-882. doi:10.1016/j.tics.2018.08.003.

Rosen, S., 1992. Temporal information in speech: acoustic, auditory and linguistic aspects. Philosoph. Trans. R. Soc. B Biol. Sci. 336 (1278), 367-373. doi:10.1098/rstb.1992.0070.

Shahin, A.J., Picton, T.W., Miller, L.M., 2009. Brain oscillations during semantic evaluation of speech. Brain Cogn. 70 (3), 259-266. doi:10.1016/j.bandc.2009.02.008.
Sheng, J., Zheng, L., Lyu, B., Cen, Z., Qin, L., Tan, L.H., Huang, M.-.X., Ding, N., Gao, J.-.H., 2019. The cortical maps of hierarchical linguistic structures during speech perception. Cerebral Cortex 29 (8), 3232-3240. doi:10.1093/cercor/bhy191.

Skipper, J.I., Devlin, J.T., Lametti, D.R., 2017. The hearing ear is always found close to the speaking tongue: review of the role of the motor system in speech perception. Brain Lang. 164, 77-105. doi:10.1016/j.bandl.2016.10.004.

ten Oever, S., Schroeder, C.E., Poeppel, D., van Atteveldt, N., Mehta, A.D., Mégevand, P, Groppe, D.M., Zion-Golumbic, E., 2017. Low-frequency cortical oscillations entrain to subthreshold rhythmic auditory stimuli. J. Neurosci. 37 (19), 4903-4912.

Teng, X., Tian, X., Doelling, K., Poeppel, D., 2018. Theta band oscillations reflect more than entrainment: behavioral and neural evidence demonstrates an active chunking process. Eur. J. Neurosci. 48 (8), 2770-2782. doi:10.1111/ejn.13742.

Tzourio-Mazoyer, N., Landeau, B., Papathanassiou, D., Crivello, F., Etard, O., Delcroix, N., Mazoyer, B., Joliot, M., 2002. Automated anatomical labeling of activations in SPM using a macroscopic anatomical parcellation of the MNI MRI single-subject brain. NeuroImage 15 (1), 273-289. doi:10.1006/nimg.2001.0978.

van Bree, S., Sohoglu, E., Davis, M.H., Zoefel, B., 2021. Sustained neural rhythms reveal endogenous oscillations supporting speech perception. PLoS Biol. 19 (2), e3001142. doi:10.1371/journal.pbio.3001142.

Vinck, M., Oostenveld, R., van Wingerden, M., Battaglia, F., Pennartz, C.M.A., 2011. An improved index of phase-synchronization for electrophysiological data in the presence of volume-conduction, noise and sample-size bias. Neurolmage 55 (4), 1548-1565. doi:10.1016/j.neuroimage.2011.01.055.

Weisz, N., Hartmann, T., Müller, N., Lorenz, I., Obleser, J., 2011. Alpha rhythms in audition: cognitive and clinical perspectives. Front. Psychol. 2. doi:10.3389/fpsyg.2011.00073.

Zatorre, R.J., Belin, P., 2001. Spectral and temporal processing in human auditory cortex. Cerebral Cortex 11, 946-953.

Zoefel, B., 2018. Speech entrainment: rhythmic predictions carried by neural oscillations. Current Biol. CB 28 (18), R1102-R1104. doi:10.1016/j.cub.2018.07.048.

Zoefel, B., Allard, I., Anil, M., Davis, M.H., 2020. Perception of rhythmic speech is modulated by focal bilateral transcranial alternating current stimulation. J. Cogn. Neurosci. 32 (2), 226-240. doi:10.1162/jocn_a_01490.

Zoefel, B., ten Oever, S., Sack, A.T., 2018. The involvement of endogenous neural oscillations in the processing of rhythmic input: more than a regular repetition of evoked neural responses. Front. Neurosci. 12. doi:10.3389/fnins.2018.00095.

Zoefel, B., VanRullen, R., 2015. Selective perceptual phase entrainment to speech rhythm in the absence of spectral energy fluctuations. J. Neurosci. 35 (5), 1954-1964. doi:10.1523/JNEUROSCI.3484-14.2015.

Zoefel, B., VanRullen, R., 2016. EEG oscillations entrain their phase to high-level features of speech sound. Neuroimage 124, 16-23. doi:10.1016/j.neuroimage.2015.08.054 